\title{
CURRENT CONCEPTS OF MORPHOLOGY OF AMORPHOUS POLYMERS- \\ II. DEGREE OF LOCAL ORDER AND OVERALL CHAIN CONFORMATION*
}

\author{
G. S. Y. YEH \\ Departments of Chemical Engineering and Materials, Metallurgical Engineoring and the \\ Macromolecular Research Center \\ The University of Michigan, Ann Arbor, Michigan 48109
}

\begin{abstract}
After a brief discussion on our earlier electron microscopy results of amorphous polymers and the results obtained by others in recent years from small angle neutron scattering and optical anisotropy measurements, it is concluded that there appears to be general agreement concerning the presence of local order and the overall statistical behaviour of macromolecules. The remainder of the discussion is on the most recent DRDF studies of a wide variety of amorphous polymers including monocrystallizable aPS, crystallizable PET and its liquid crystalline copolymer $40 \mathrm{PET} / 60 \mathrm{PHB}$, PC, NR melt, PE melt and amorphotized PE. It is shown that DRDFs, when analysed properly and in detail, e.g. by means of application of the paracrystalline lattice theory, can provide information about (1) the origin of the DRDF peaks, (2) the extent of local order, and (3) the effects of additional ordering by annealing, chain stiffening and intermolecular crosslinking.
\end{abstract}

\section{INTRODUCTION}

In recent years, the question of local order and overall chain conformation in amorphous, noncrystalline polymers has been raised and discussed on numerous occasions at international conferences. This is the second time I have been invited to speak on the subject at an IUPAC meeting; the first time was in 1971 in Prague (Part I [1]). At that time I discussed our findings of a nodular microstructure in typical amorphous polymers, including the diffraction microscopy evidence for ordered domains consisted of parallel chain segments. I also discussed the importance of nodules and ordered domains in polymer deformation and crystallization. For example, the nodules can be seen to play a direct role in the formation of chain-folded lamellae upon annealing of isotactic polystyrene from the glass (Fig. 1) or upon cupercooling natural rubber from the melt (Fig. 2). Perfection of order can be shown to take place locally. This is indicated by the gradual increase in contrast (Figs. 1 and 2) between the crystallizing nodules and the "amorphous" matrix. However, the overall conformation of the mole-

* Vysokomol. soyed. A21: No. 11, 2433-2446, 1979. 


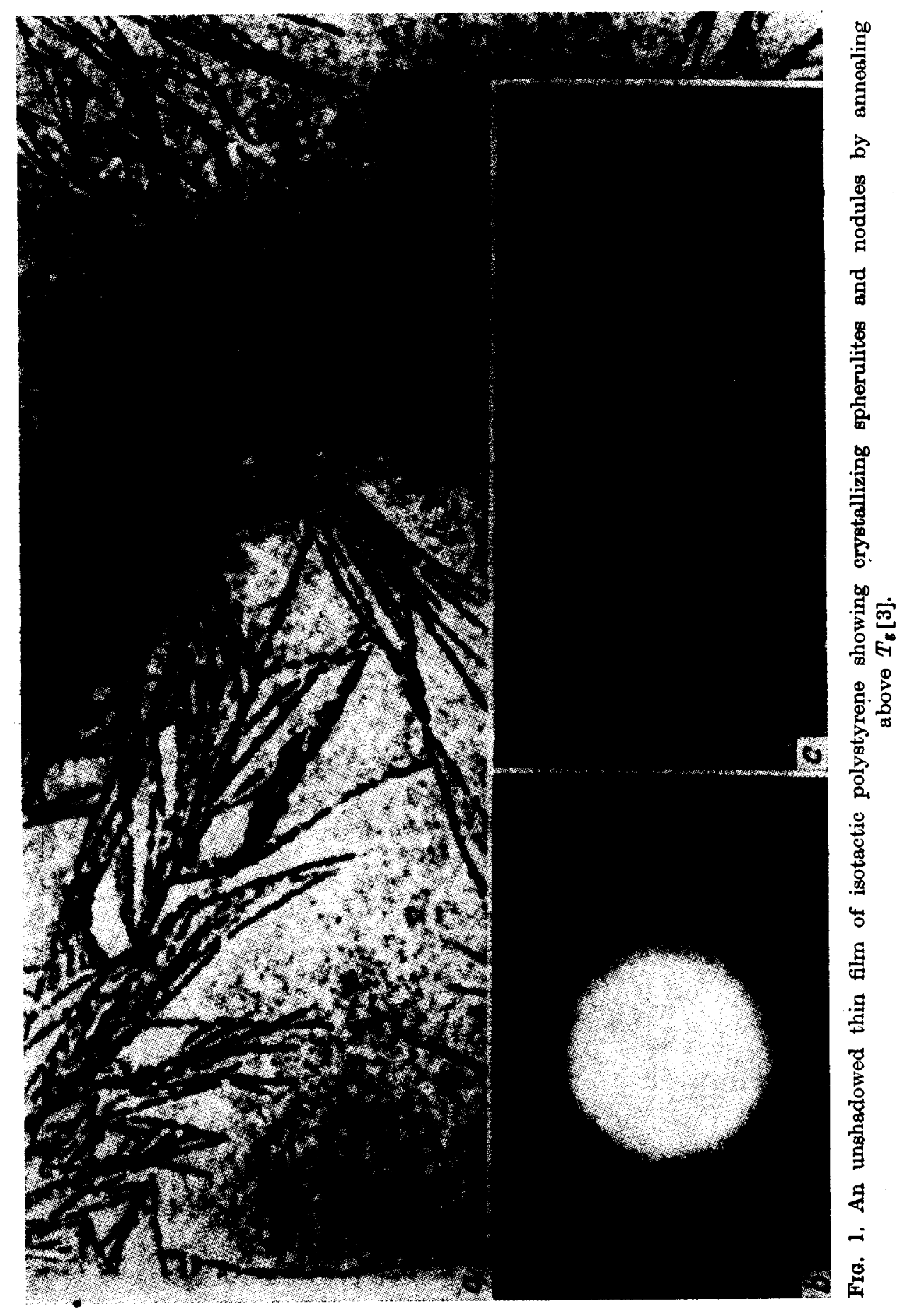


cule, as indicated by the dotted line in the Folded-Chain Fringed-Micelle Grain model (Fig. 3 [2]), remains essentially the same as in the amorphous glass or molten state [3]. Recent small angle neutron scattering (SANS) results [4-8] of molten and crystallized polyethylene, polypropylene and polyethylene oxide showing the same overall "random coil" behaviour of macromolecules in both

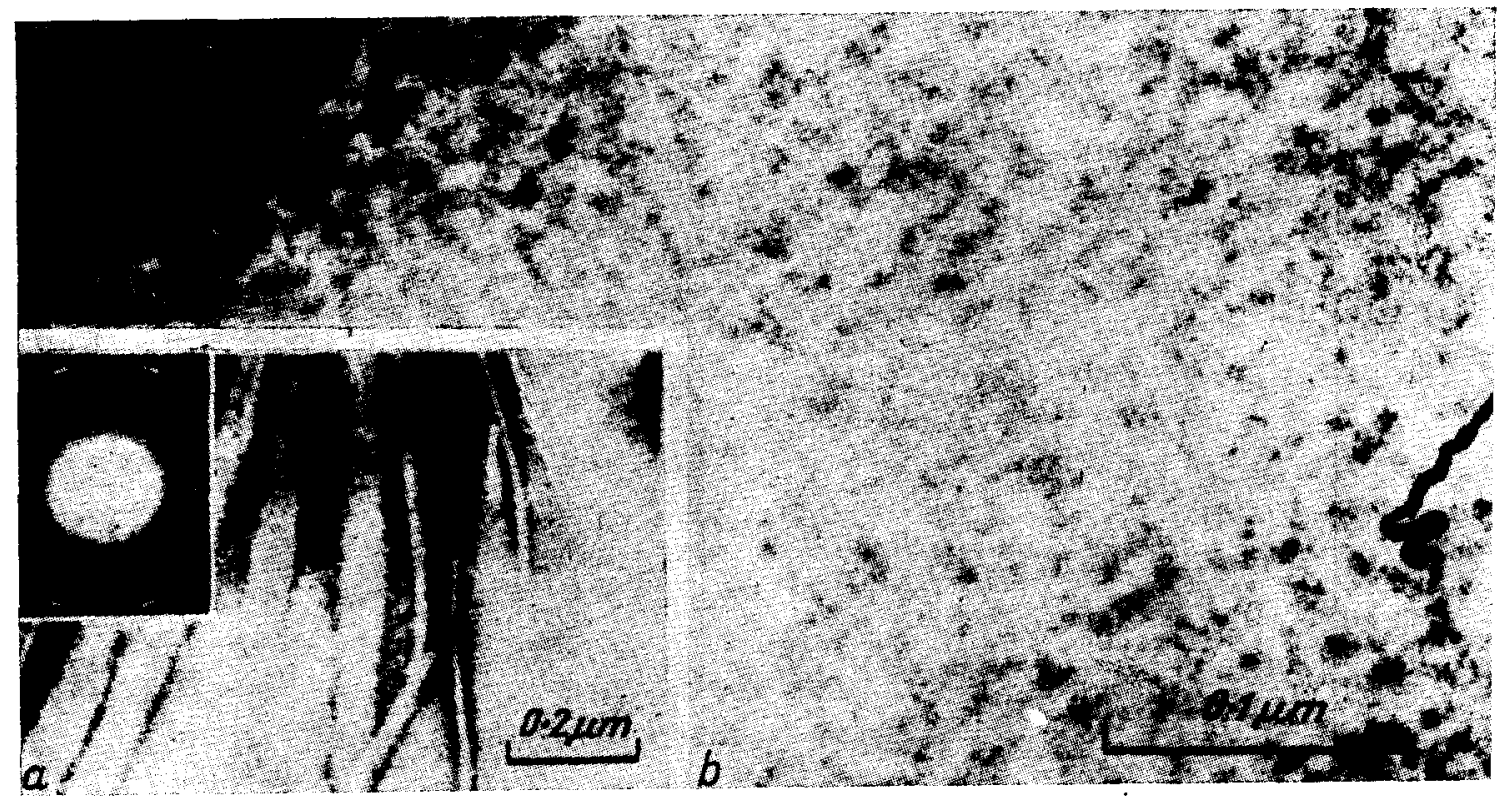

Fig. 2. An unshadowed $\mathrm{OsO}_{4}$ stained thin film of $\mathrm{NR}$ showing growing lamellae from a crystallizing spherulite $(a)$ and nodules $(b)$ undergoing a transition from a less ordered to a more ordered state [31].

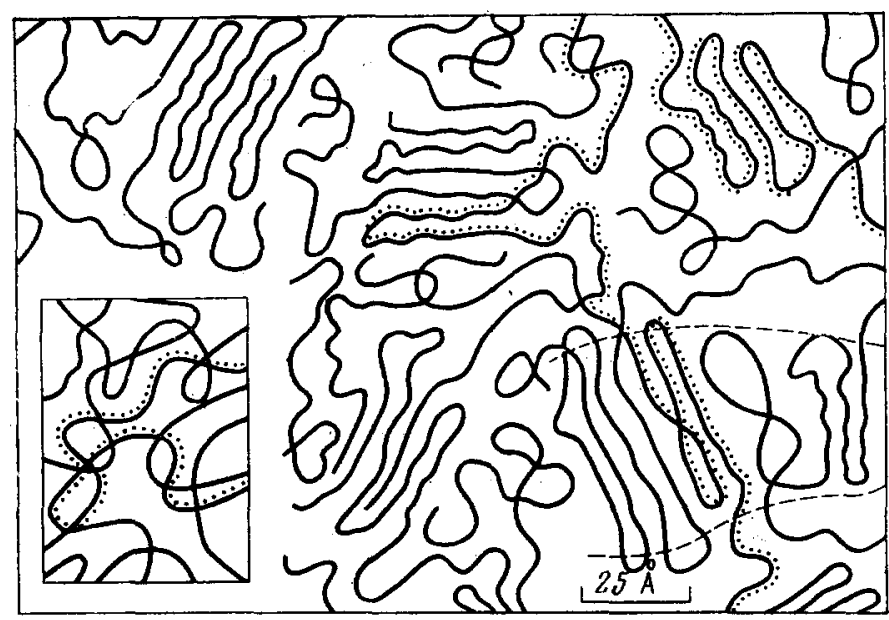

Fic. 3. Structural model for the amorphous state of polymers [21]. 
states can be adequately accounted for by the structural model in Fig. 3 for both the noncrystalline and the crystallized states $[2,3]$.

This discussion (Part II) will be concentrating primarily on our most recent wide angle X-ray scattering (WAXS) studies of some of the most typical noncrystalline polymers. WAXS, being another direct structural technique for revealing local atomic positions, should provide similar, but generally more quantitative information, to the information revealed by diffraction microscopy. In particularlar, we are interested in establishing a suitable experimental method for quantitatively determining the type and the extent (degree) of local (short range) order and how it depends on polymer type, physical state, degree of crystallization or chain rigidity, and intermolecular crosslinking. The experimental method we have chosen to refine is WAXS analysis by means of differential radial distribution function. The results will be discussed after a few brief comments regarding the extent of agreement or disagreement on the presence of local order in the noncrystalline states of polymers.

\section{ORDER VS DISORDER IN AMORPHOUS POLYMERS}

As I pointed out in Kyoto [9] that in spite of the apparent controversy on the subject, there appeared, at least from the experimental point of view, very little disagreement regarding (1) the overall Gaussian behavior of macromolecules in their amorphous glass or melt states and (2) the presence of order at the local level. For example, the overall Gaussian behavior of the macromolecule was retained by us not only in the amorphous, but also in the crystallized state, as shown by the dotted macromolecules in Fig. 3. This deduction is also indicated by recent SANS results on molten and crystallized polymers [4-8]. The extent of local order is limited as shown by diffraction microscopy for molten natural

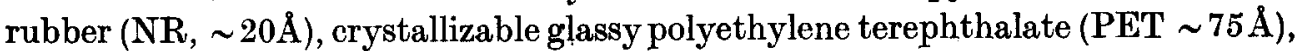
and noncrystallizable atactic polystyrene (aPS, $\sim 30 \AA$ ). Evidence of local order (lateral correlation) has also come from optical anisotropy measurements [10-11], including those from Flory's own group [12], indicating that the order in polymers is greater than corredponding low molecular weight materials. Furthermore if one takes into consideration the fact that neither calculated chain dimensions based on rotational isomeric state approximations [13] nor measured chain dimensions of amorphous or crystallized polymers based on SANS [4-8] are very sensitive to the presence of all trans sequences (up to as many as 20-40 in a single stretch), any remaining disagreement regarding the presence of local order will probably disappear in time.

The major issues which we had raised in 1971-1972 [1-2] were (and still are) (1) what is the type and extent of local order in a given amorphous polymer, and (2) what is the extent of chain folding (due possibly to chain coiling) for a given polymer at a given condition. DRDF analysis of WAXS can provide some of the answers, especially regarding the first question. For example, one should expect in the DRDF curves intermolecular periodicities (peaks) corresponding 
to the more or less fixed distances between atoms in neighbouring parallel chain segments as opposed to the absence of such intermolecular peaks in a truly random case (insert in Fig. 3). To determine the extent of intramolecular chain folding one has to use wide angle neutron scattering (WANS) of tagged single molecules in bulk and such experiments have yet to be performed.

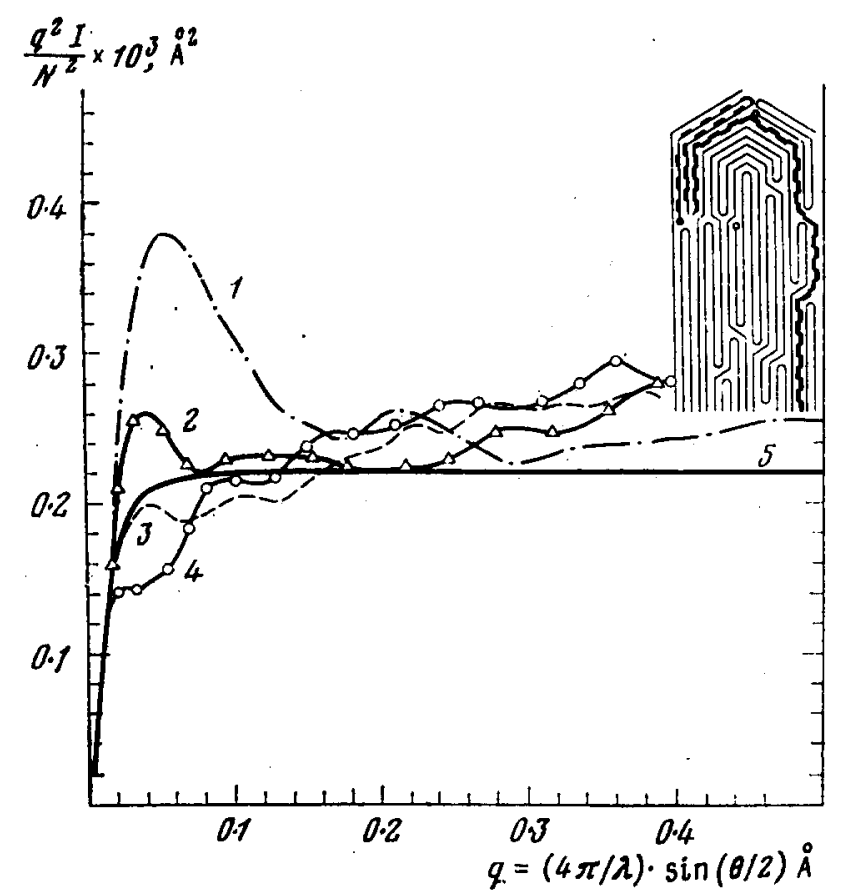

FIG. 4. Theoretical vs. experimental curves of $I q^{2} / N^{2}$ vs. $q$ for molten PE [14].

Nevertheless, it is of interest to note that Pechhold et al. [14] had recently introduced the adjacent chain folding idea into his model calculations. Using reasonable fold energies they were able to synthesize theoretical plots for both molten polyethylene (PE) and noncrystallizable aPS that became in close agreement with the experimental curves, as shown in Fig. 4 for PE.

\section{WAXS AND DRDF}

WAXS, besides diffraction or direct imaging electron microscopy, is probably the only other reasonably well-established structural technique close to being able to provide direct information about chain packing in amorphous polymers. (One can in theory employ neutrons or electrons at wide angles as well but the accuracies are generally less). The structural information is usually derived from the RDF or the DRDF (or the so-called differential pair correlation function), $4 \pi r^{2}[\rho(r)-\bar{\rho}]$. The DRDF represents the density of atoms $\rho(r)$ above the mean density $\bar{\rho}$ in a spherical shell of thickness $d r$ at a distance $r$ from an arbitrarily 
chosen reference atom. It is related to the interference function $i(s)$ by Fourier transformation:

$$
4 \pi r^{2}[\rho(r)-\bar{\rho}]=4 \pi r^{2} \bar{\rho} H(r)=\frac{2 r}{\pi} \int_{0} s i(s) \sin s r d s,
$$

where $s=\frac{4 \pi}{\lambda} \sin \theta, 2 \theta$ is the scattering angle, $\lambda$ is the wavelength of the incident beam and $i(s)=\frac{I(s)}{f^{2}}-1$, where $f$ is the scattering factor for the atom in a monoatomic scattering system [15]. For a multi-atomic system $f_{i}$ 's and different definitions for $H(r)$ and $i(s)$ will have to be introduced [16].

$I(s)$ can be obtained from the raw scattering data after suitable corrections for background scattering, polarization, absorption, inelastic scattering and multiple scattering, and after proper normalization to absolute units and subtraction of incoherent Compton scattering. Our raw data [15, 16] were generally collected on a Phillips Norelco diffractometer employing a monochromatized $\mathrm{Cu} K_{\alpha} 1.54 \AA$ radiation. A pulse height discriminator was set to accept $90 \%$ of the total intensity and the background noise was cut down to less than 0.1 count per second. Data were collected by step scannings with $0.2^{\circ}$ intervals from $6^{\circ}(2 \theta)$ to $45^{\circ}, 0 \cdot 5^{\circ}$ intervals from $45^{\circ}$ to $75^{\circ}$, and $1^{\circ}$ intervals from $75^{\circ}$ to $145^{\circ}$.

Normalization to the electron units (EU) was carried out using the conservation of energy and/or the limit method to get the most accurate normalization constant. Errors introduced in the normalization constant can give rise to oscillations below $\sim 1 \AA$ in the resultant DRDF curve.

The DRDF was then derived from $i(s)$ by means of Fourier transformation as indicated earlier. One major source of error involved in the calculation is the so-called termination error since instead of an infinite $s$ in the desired integration, experimental $s$ reaches up to $7 \cdot 77 \AA^{-1}$ only for $\mathrm{Cu} K_{\alpha}$ radiation. This type of error can easily be detected by the ripples superimposed on the DRDF mains peaks at $r \sim 3$ to $7 \AA$. In our studies, the elimination of these ripples is carried out by a number of extensions of the $s i(s)$ vs. $s$ plot to $s=16 \AA^{-1}$ through iterations of transformation and inverse transformation.

Another major source of error in the DRDF construction is due to the published values of scattering factors which can cause oscillation in the region below $\sim 1 \AA$ of the DRDF. These are eliminated in our studies using the procedure described by Kaplow and co-workers. Please refer to our earlier publications for details of proper construction of DRDF curves [15-16].

\section{DRDF ANALYSIS}

Many of our experimental DRDF curves have been subjected to three methods of analysis to determine the origin(s) of DRDF peaks:

(1) By comparison with peak intensities and peak locations in the DRDF' of corresponding crystallized samples one can obtain some information about the 
origin of peaks (intra vs. intermolecular) and whether the peaks arișe from some local ordering or not. Presence of local ordering should lead to minimal changes in peak positions upon crystallization from those already present in the amorphous state.

(2) Separation method. Briefly in the separation method contributions of intramolecular atomic distances to the experimental DRDF are calculated from an assumed chain conformation, usually of a crystalline type to give maximum . contributions of fixed intramolecular atomic distances. The calculated values are

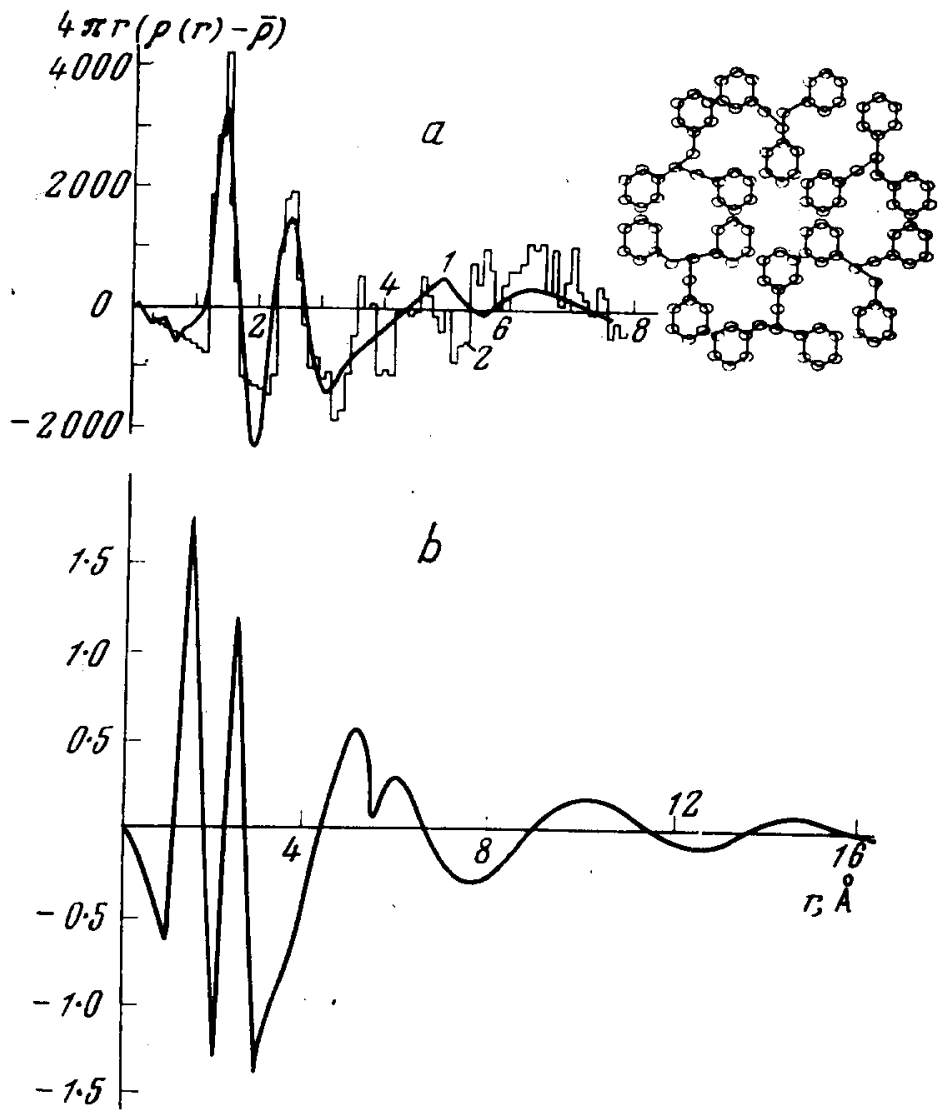

Fig. 5. Top. $4 \pi r[\rho(r)-\bar{\rho}]$ vs. $r$ [19], showing experimental (full curve) vs. theoretical (histogram) DRDF. The latter is based on the unit cell of crystalline iPS projected onto an (001) plane indicated on the right. Bottom. $4 \pi r[\rho(r)-\bar{p}]$ vs. $r$ for quenched aPS obtained by Gupta and Yeh [16].

then compared with the experimental findings based on measured areas from the RDF curves to separate the intra from the intermolecular contributions. The method was first employed by Bjorhnaug et al. [17] and more recently by Wignall and Longman [18] and by us [15, 16]. Wecker et al. [19] have also used a similar method, but instead of separating the intermolecular contributions by subtrac- 
tion, they calculated both intra and inter chain contributions by assuming a hexagonal crystalline chain packing for isotactic PS to get the total DRDF and compare it with the experimental DRDF (see Fig. 5 top).

The above two methods are relatively simple and usually the only ones used in the analysis of DRDFs of polymers. However, both methods have one major drawback, namely they do not provide direct evaluations of intermolecular contributions to the experimental peaks.

(3) Application of the paracrystalline lattice theory. This method can provide directly not only information about lateral ordering but also information about the structure, i.e. the atomic positions (in theory at least) in a distorted lattice. The method has been successfully applied to molten metals by Hosemann and coworkers [20] and more recently to amorphous PE by Gupta and Yeh [21]. The theory describes the structure by a convolution polynomial made up of first neighbour statistical distributions. The paracrystalline lattice planes are displaced and the long range order of the system is lost eventually. Lattice distortions of this type are known as distortions of the second kind. The well known distortions of the first kind, on the other hand, preserve the long range order of the materials. To date only the theory of ideal paracrystals has been applied to metals [20] or polymers [21] and such applications are already very complicated for simple polymers like polyethylene. For a given polymer the fitting between the experimental and the theoretical paracrystalline DRDF is very sensitive to proper choice of lattice, lattice parameters and distortion parameters [21].

Briefly, if the first-neighbour distance statistics $H_{1}$ are known, then the distance statistics between second neighbours and further statistics $H_{i}$ are calculated by repeated convolutions of $H_{1}$ with $H_{1}$. Thus

$$
H_{i}=H_{1}^{*} H_{1}^{*} \ldots H_{1}(i-1) \text { times }
$$

where ${ }^{*}$ denotes the convolution operation:

$$
\begin{gathered}
H_{1}(\mathbf{r}) * H_{1}(\mathbf{r})=\int_{0}^{\infty} H_{1}(\mathbf{y}) \cdot H_{1}(\mathbf{r}-\mathbf{y}) d \mathbf{y} \\
\int_{0}^{\infty} H_{1}(\mathbf{r}) d^{3} \mathbf{r}=1
\end{gathered}
$$

In this way a synthetic RDF, $4 \pi r^{2} \rho(r)$, can be generated from lattice distances containing lattice distortions of the first and the second kind:

$$
4 \pi r^{2} \rho(r)=H_{1 \text { st kind }} *\left[\sum k_{i} H_{i}\left(\mathbf{r}-\mathbf{r}_{i}\right)\right]
$$

where $\rho(r)$ is now $Q(r)$, the so-called $Q$-function $[20,21]$, divided by $N$ (i.e. $\frac{Q(r)}{N}$ $\left.\equiv \rho(\bar{x})^{*} \rho(-\bar{x})\right), k_{i}$ and $r_{i}$ are the coordination number and the center of $i$-th distance statistics. The synthetic DRDF was obtained by substracting $4 \pi r^{2} \bar{\rho}$ from the synthetic RDF. 


\section{DRDF RESULTS}

The results from our most recent DRDF studies [15-16, 21] of a number of noncrystalline polymers will be summarized and discussed. The emphasis will be on (a) quality and reproducibility of the DRDF, (b) origin of the DRDF peaks, (c) extent of lateral ordering, and (d) wherever appropriate, comments on the effects of additional ordering by annealing, chain stiffening and intermolecular crosslinking.

1. Non-cryttallizable atactic polystyrene $(a P S)$. As one of the most typical amorphous polymers, the extent of lateral ordering has been examined by many different techniques [1] including the most recent application of SAXS by Harget and Aharoni [22] showing inhomogeneities $\sim 30 \AA$ in aPS and the recent application of DRDF by Wecker et al. [19] who concluded "the results of this study indicate that a short range structure exists in noncrystalline PS caused by a tendency for phenyl rings to separate and chain segments to pack parallel to each other".

Their results [19] on two glassy aPS $\left(\rho=1.048 \mathrm{gm} / \mathrm{cm}^{3}\right)$ and two quenched isotactic PS $(\rho=1.056)$ showed almost identical DRDFs with major peaks at $1 \cdot 5$, 2.5, 5, 6 and $10 \AA$ plus a weaker one at $15 \AA$. This indicates to us that whatever order is present in quenched crystallizable iPS is most likely present in the noncrystallizable aPS. They were able to identify the origins of the various DRDF peaks in a partially crystallized iPs $(\rho=1.069)$ from comparison with a theoretical DRDF hostogram that shows the contributions of various intra and intermolecular distances present in an iPS crystalline lattice (Fig. 5). By comparing the locations and the intensities of the intermolecular peaks at $r>6 \AA$ with those in the DRDFs of noncrystalline PS, they reached the conclusion, quoted earlier, that there is a short range structure in aPS.

Figure 5 also shows an example of a DRDF for a quenched aPS obtained recently in our laboratory [16]. In terms of peak height, peak location and overall peak profle, it is identical to the ones reported by Wecker et al. for aPS and shows the reproducibility of DRDF curves when proper considerations are given to the WAXS data correction and DRDF construction. The DRDF peak at 10 arises from parallel chain packing and is believed to contribute to the intermolecular 9.3 $\AA d_{\text {Bragg }}$ spacing seen in the $I$ vs. $2_{\theta}$ plot. This Bragg spacing was first suspected by Katz [23] to be caused by aligned chain segments in amorphous PS. The DRDF shows average lateral correlations of $r \sim 15 \AA$, or about the size of an ordered domain seen in dark field electron microscopy [24], but smaller than the $30 \AA$ nodules see in replicas [24].

2. Glassy polyethylene terephthalate $(P E T)$ and annealed PET. The first clear evidence of lateral ordering in amorphous polymers was given for unshadowed thin films of PET using bright field and dark field electron microscopy [25]. To complement these earlier microscopy studies we decided to apply our perfected DRDF analysis to WAXS of glassy PET $\left(\rho=1.335 \mathrm{gm} / \mathrm{cm}^{3}\right)$ and PET after additional ordering by annealing at $190^{\circ} \mathrm{C}$ for $15 \mathrm{~min}(\rho=1.364)$ and $30 \mathrm{~min}$ 
$(\rho=1.385)$ [16]. We found that the corrected intensity profile $(I \mathrm{vs} 2 \theta)$ of the glassy PET shows three peaks, one being intermolecular at $d_{\mathrm{Bragg}}=4 \cdot 19 \AA$ and the other two being intramolecular at $d=2.13$ and $1.19 \AA$. The sample annealed for 15 min at $190^{\circ} \mathrm{C}$ shows splitting of the $4.19 \AA$ peak into 3 peaks at $3.50 \AA$ $\left\langle\left(d_{100}\right), 3.96 \AA\left(d_{1 \overline{1} 0}\right)\right.$ and 5.21 (mainly $\left.d_{010}\right)$. The presence of only $h k o$ type reflections

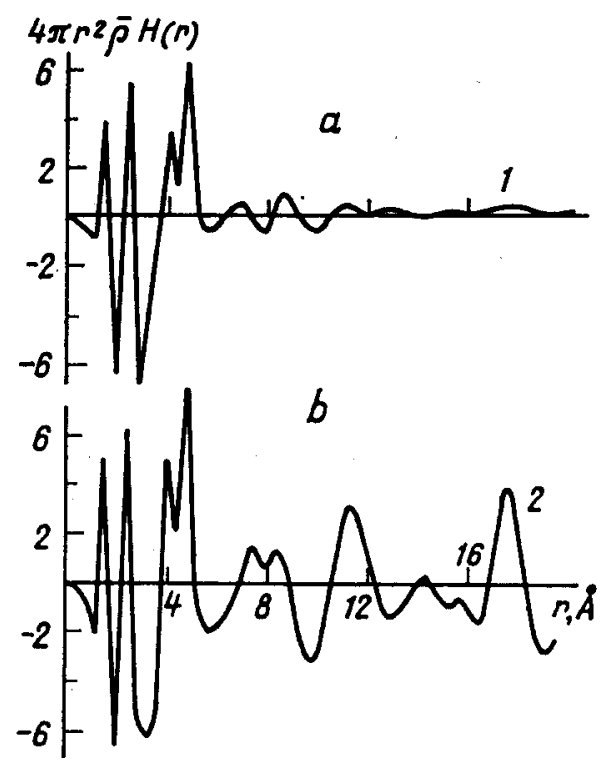

FIG. 6. DRDFs for glassy PET (a) and annealed PET $(b)$ [16].

indicates that this sample is still not yet fully crystallized and will be briefly discussed here together with the results on glassy PET. Longer annealing for $\mathbf{3 0}$ min at the same temperature produces two additional peaks at $4 \cdot 11 \AA\left(d_{\overline{11}}\right)$ and $5.49 \AA\left(d_{01 \overline{1}}\right)$, indicating that crystallization has occurred.

The DRDF of all three samples are similar in some aspects, all showing peaks at $1 \cdot 50,2 \cdot 45,4 \cdot 15,4 \cdot 90,6 \cdot 4,8 \cdot 5,12 \cdot 0$ and $17 \cdot 7 \AA$. However, the annealed samples shows more. For example, comparing to the glassy PET, the sample annealed for 15 min shows that the weak peaks seen in glassy PET at and above $4.9 \mathrm{~A}$ become more prominent and slightly shifted to lower $r$ values (Fig. 6). It suggests that these peaks at and above $4.9 \AA$ are intermolecular in origin in glassy PET and are the consequence of more or less aligned segments which upon annealing become better aligned. Furthermore, additional peaks are seen at 1·4, 17·6, 21·6, 24.4 and 28.3 A. Similar results were obtained for crystallized PET after annealing at $190^{\circ} \mathrm{C}$ for $30 \mathrm{~min}$. Thus the process of crystallization is a local one, unlike what has generally been assumed in a chain folding type crystallization process, with the molecules being reeled in and chain folded one at a time from an entirely random state. It is, however, in agreement with our proposed crystallization process [3]. 
The assignments of intermolecular peaks at and above $4.9 \AA$, based on crystal lization studies, are verified by calculations based on the separation method [16]. As seen in Table 1, the area for the experimental peak at $4.9 \AA$ is about 7 times greater than the calculated value of the intramolecular peak at $4.86 \AA$, showing that $\sim 80 \%$ of the peak is due to intermolecular contributions in the glassy PET.

Table 1. Comparison of calculated intrachain distances and their peak areas: UNDER THE RDF CURVE WITH THE EXPERIMENTAL PEAKS AND THEIR AREAS

\begin{tabular}{|c|c|c|c|c|c|c|c|}
\hline \multicolumn{2}{|c|}{ Calculated peaks } & \multicolumn{6}{|c|}{ Experimental peaks } \\
\hline \multirow{2}{*}{$\begin{array}{c}\text { peak, } \\
r, \AA\end{array}$} & \multirow{2}{*}{$\begin{array}{l}\text { area, } \\
\text { atoms }\end{array}$} & \multicolumn{2}{|c|}{ glassy PET } & \multicolumn{2}{|c|}{$\mathrm{PET}, 190^{\circ}, 15 \mathrm{~min}$} & \multicolumn{2}{|c|}{ PET, $190^{\circ}, 30 \mathrm{~min}$} \\
\hline & & $r, \AA$ & area & $r, \AA$ & area & $r, \AA$ & area \\
\hline $1 \cdot 46$ & 3.08 & 1.50 & $2 \cdot 89$ & 1.45 & $2 \cdot 98$ & $1 \cdot 40$ & $3 \cdot 13$ \\
\hline $2 \cdot 45$ & 5.96 & $2 \cdot 45$ & $5 \cdot 93$ & $2 \cdot 40$ & $5 \cdot 94$ & $2 \cdot 35$ & 6.02 \\
\hline $3 \cdot 67$ & $2 \cdot 18$ & $4 \cdot 15$ & $5 \cdot 49$ & 4.05 & $5 \cdot 75$ & $4 \cdot 00$ & $6 \cdot 05$ \\
\hline $4 \cdot 15$ & 1.93 & $4 \cdot 15$ & $5 \cdot 49$ & 4.05 & $5 \cdot 75$ & 4.00 & 6.05 \\
\hline \multirow[t]{2}{*}{$4 \cdot 68$} & 3.02 & $4 \cdot 90$ & $21 \cdot 45$ & $4 \cdot 85$ & $23 \cdot 80$ & $4 \cdot 80$ & $26 \cdot 60$ \\
\hline & & & & & & $5 \cdot 70$ & $8 \cdot 00$ \\
\hline
\end{tabular}

Based on these two analyses we conclude that the lateral order in glassy PET extends to $r \sim 17.7 \AA$. As expected, it is smaller than the average size of nodules. $(\sim 75 \AA)$ seen in replicas, which may consist of several ordered domains.

Earlier, Longman et al. [26] had also reported some DRDF studies of a glassy PET' (and three samples annealed for $100 \mathrm{hr}$ at 65,80 , and $90^{\circ} \mathrm{C}$ ). There is general agreement between the two DRDFs of glassy samples. However, the oscillations below 1.0 $\AA$ are present in their DRDFs. In addition, their DRDF showed peaks at $1.92,2 \cdot 86$, and $3.68 \AA$ which we believe are caused, at least partly, by errors due to termination, because they are removed in our DRDF, especially in view of the fact that these peaks are located at distances of $0.48 \pm 0.02 \AA$ from the real peaks at $1 \cdot 43,2 \cdot 40$ and $4 \cdot 15 \AA$. The distance of $0 \cdot 48 \AA$ is the period of the ripples, i.e. $7 \cdot 8 / S_{\max }$.

3. Liquid cryttalline 40 polyethylene terephthalate/60 poly-p-hydroxybenzoic acid (40 $P E R / 60 P H B$ ). Based on the finding that longer range local order can be seen in annealed PET with some straightening of the molecular chain segments, we decided to examine the effect of chain straightening by chemical means using a PET copolymerized with various amounts of PHB monomers [27]. By incorporating proper amounts of $\mathrm{PHB}$ units, PET can be made to exhibit thermotropic liquid crystalline characteristics [28] such as low melt viscosities and optical birefringence. We have chosen a $40 \mathrm{PET} / 60 \mathrm{PHB}$ as an example to show how such liquid-crystalline features can also be reflected in changes in its corresponding DRDF (Fig. 7). Compared to the DRDF for glassy PET (Fig. 6), it now has all prominent peaks, located at $1 \cdot 55,2 \cdot 40,5 \cdot 0,9 \cdot 9,14 \cdot 2,18 \cdot 9,23 \cdot 2,27 \cdot 5$, and 
31.7 \&. The first two relatively sharp peaks in the DRDF are due to the average first and the average second nearest intramolecular atomic distances. The remaining peaks, as in the case of PET, are predominantly intermolecular in origin, as judged by the presence of a nearly constant periodicity of about $4.5 \AA$ in the DRDF. The periodicity is attributed to the nematic ordering of neighbouring chain segments. Thus, in the $40 \mathrm{PET} / 60 \mathrm{PHB}$ copolyester a liquid crystalline order with $r \sim 32 \AA$ is also deduced from DRDF analysis. It is about 14-15 $\AA$ greater than glassy PET using the same DRDF criterion. Therefore it appears

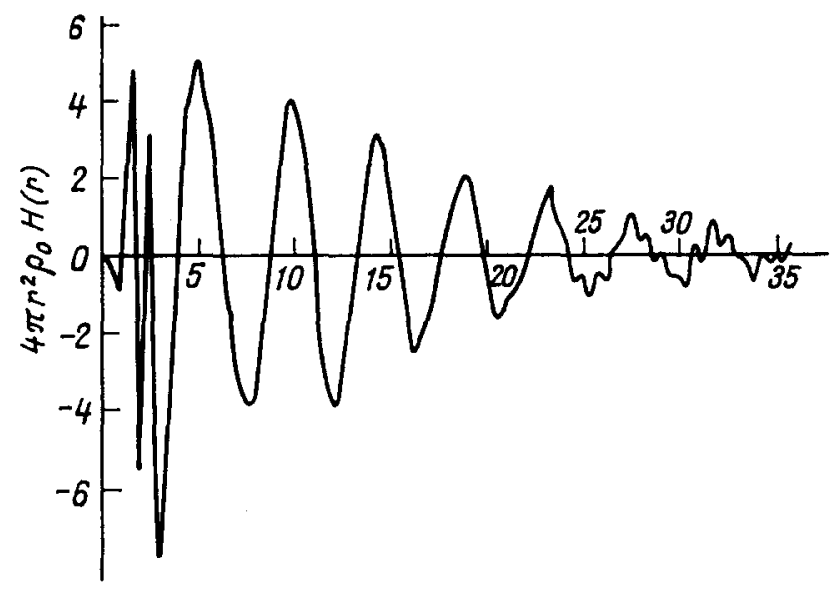

FIG. 7 DRDF for the liquid crystalline 40 PET/60 PHB copolyester [27].

that lateral ordering in amorphous polymers can be substantially improved by modification of the chemical configuration of macromolecules. In the copolyester, the improvement is brought about by incorporating more of the planar parasubstituted PHB moieties.

'4. Glassy polycarbonate $(P C)$. With a repeat unit consisting of one more benzene ring than PET, PC is a slightly more rigid polymer, but certainly not as rigid as the liquid crystalline $40 \mathrm{PET} / 60 \mathrm{PHB}$ described earlier. $\mathrm{PC}$ is also of interest because in the $I$ vs. $2 \theta$ plot there are usually two intermolecular peaks, [24] one located at $d_{\mathrm{Bragg}}=4.9 \AA$ corresponding approximately to $d_{020}$ spacings and the other at $d_{\mathrm{Bragg}}=11.5 \AA$ corresponding approximately to combinations of $d_{100}(11.9 \AA)$ and $d_{010}(10 \cdot 1 \AA)$ spacings.

There are two DRDFs published in the literature for glassy $\mathrm{PC}$, one by Wignall and Longman [18] who concluded that local ordering does not extend beyond $r \sim 10 \AA$ and a slightly improved one by us [16]. Ours is shown here with peaks located at $1 \cdot 5,2 \cdot 5,5 \cdot 05,6 \cdot 5,11 \cdot 3,17 \cdot 4,22 \cdot 7$ and $28.5 \AA$ (Fig. 8). (Wignall and Longman reported that the first intermolecular peak is located at 5.5 $\AA$. However, we measured the value from their DRDF and found that $r$ at $6.5 \AA$ has maximum peak height). Thus the main difference in our DRDF is that we see interchain peaks from $6 \cdot 5$ to $28 \AA$ with the period of $\sim 5 \cdot 5 \AA$, caused by lateral chain packin $g$ - 
'The order is about 11 \& greater than the size detected in PET by the same criterion, and much smaller than the nodules reported in the literature for PO by -SAXS or EM [1], which as mentioned earlier may consist of several ordered domains.

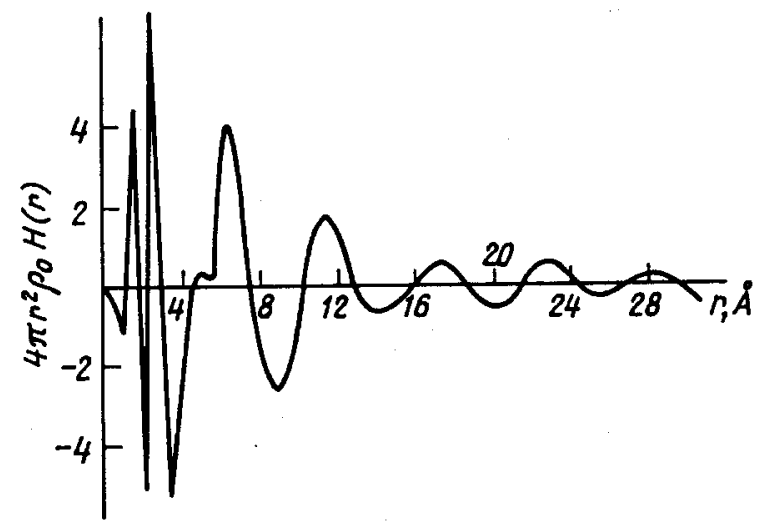

Fig. 8 DRDF for a glassy PC [16].

5. Natural rubber $(N R)$ melt. Being a melt at room temperature, NR has been - favorite candidate to examine presence of order in molten polymers. In fact the first application of the DRDF method to polymers was on NR, being carried out by Simard and Warren in 1936, and the results were discussed in the 1972 review [1]. No consideration of lateral order was given in this very early work.

In 1972 Bokhyan and co-workers [30] reported another DRDF on NR and showed three broad peaks at 2, 4.5-8 and 10-12 $\AA$ (Fig. 9). The first peak was attributed to combination of distances of $\mathrm{C}_{1}-\mathrm{C}_{2}$ and $\mathrm{C}_{1}-\mathrm{C}_{3}^{-}$, and the remaining two peaks were considered to be mainly intermolecular in origin. Based on sample density and intermolecular peak position considerations, they postulated an orthorhombic packing arrangement for the NR molecules in the melt.

In 1978 [15] we reported a much improved DRDF for NR and which is also shown here in Fig. 9 to compare with the one obtained by Bokhyan and coworkers. It contains six peaks located at $1.5,2 \cdot 48,3.98,5 \cdot 68,10 \cdot 65$ and $15.24 \AA$. Using the separation method (see results in Table 2) we were able to establish that the first two peaks are nearly $100 \%$ intramolecular and the third peak is about $50 \%$ intramolecular in origin. The intramolecular origin of the fourth peak can be assigned to the combination of $\mathrm{C}_{1}-\mathrm{C}_{6}$ and $\mathrm{C}_{1}-\mathrm{C}_{7}$ intramolecular atomic distances, giving rise to an average atomic distance of $5.89 \AA$ which matches well with the experimentally observed position at $5 \cdot 68 \AA$. The intramolecular contribution to this fourth RDF peak area decreases abruptly to $10 \%$ and this -suggests that this peak is mainly intermolecular. Based in a similar estimate, the remaining peaks must then originate from intermolecular distances since as the radius goes higher, experimental areas of these peaks increase much faster than the calculated intramolecular contributions. The intermolecular peaks at 
$5 \cdot 68,10 \cdot 65$ and $15 \cdot 25 \AA$ show an approximately constant periodicity of about $5.26 \AA$ which we suggest is due to the distance between neighboring parallel chain segments contained in the $130 \AA$ size nodules [31]. Application of Hose-

Table 2. Comparison of EXPerimental and MaIN Calculated INTRAMOLECULAR RDF PEAK AREA

\begin{tabular}{|c|c|c|c|c|}
\hline \multicolumn{2}{|c|}{ Experimental RDF peaks } & \multicolumn{3}{|c|}{ Calculated intramolecular peaks } \\
\hline$r, \AA$ & $\begin{array}{l}\text { area, } \\
\text { atoms }\end{array}$ & $r, \AA$ & $\begin{array}{l}\text { area, } \\
\text { atoms }\end{array}$ & $\frac{\text { area }}{\text { arealc }}$ \\
\hline $1 \cdot 51$ & $1 \cdot 88$ & $1 \cdot 47^{\mathrm{b}}$ & $2 \cdot 0$ & 1.06 \\
\hline $2 \cdot 48$ & $2 \cdot 52^{\mathrm{c}}$ & $2 \cdot 49^{\mathrm{d}}$ & $2 \cdot 4$ & 0.95 \\
\hline 3.98 & $9 \cdot 01^{\mathrm{c}}$ & $3.97^{\mathrm{e}}$ & $4 \cdot 8$ & 0.53 \\
\hline $5 \cdot 68$ & $54 \cdot 52^{p}$ & $5 \cdot 89^{\mathrm{g}}$ & $5 \cdot 2$ & $0 \cdot 10$ \\
\hline
\end{tabular}

The calculation was based on the chain conformation of crystalline NR.

b Average of all possible intramolecular $\mathbf{C}_{1}-\mathbf{C}_{2}$ distances.

c Peaks were resolved following the general slope of the RDF curve on their left-nand side and were constructed symmetrically on their right-hand side if necessary.

- Average of all possible intramolecular $\mathrm{C}_{1}-\mathrm{C}_{3}$ distances.

- Average of all possible intramolecular $\mathrm{C}_{1}-\mathrm{C}_{4}$ and $\mathrm{C}_{1}-\mathrm{C}_{6}$ distances.

: RDF peak area was estimated by summing up DRDF peak area and $f 4 \pi r^{2} \rho_{0} d r$ with its integration boundaries, the same as the two boundaries of the corresponding DRDF peak along the $r$ axis.

Average of all possible intramolecular $\mathrm{C}_{1}-\mathrm{C}_{8}$ and $\mathrm{C}_{1}-\mathrm{C}_{7}$ distances.

mann's paracrystalline theory, to the DRDF is now being carried out in our laboratory. So far our fitting suggests that a highly distorted orthorhombic unit cell is being retained in the molten state of $\mathrm{NR}$ [32].
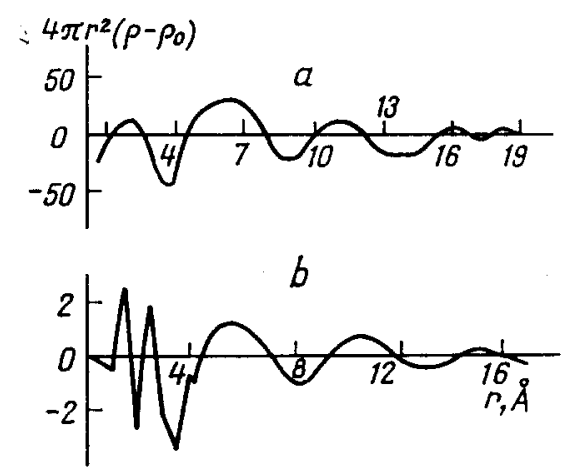

Fig. 9. DRDFs for NR [15].

6. Polyethylene (PE) melt and amorphotized polyethylene (APE). The DRDF analysis has been applied to wide angle electron diffraction (WAED) of molten PE [33]. As discussed in that 1972 review, Ovchinnikov et al. [34] reported DRDF peaks at $1 \cdot 6,2 \cdot 5,3 \cdot 2,5,10$ and $15 \AA$ for $\mathrm{PE}$ at $145^{\circ} \mathrm{C}$. The first three were attributed to be intramolecular and the rest were intermolecular. With increasing 
temperatures (up to $211^{\circ} \mathrm{C}$ ) the intermolecular peaks became more diffuse. They compared the intermolecular peaks with an assumed array of hexagonally packed chain segments. On the basis of this comparison the authors proposed that molten PE consisted of ordered regions $\sim 50 \AA$ in diameter.

However, the conclusion that parallel chain packing occurs in molten PE was challenged by Voigt-Martin and Mijlhoff [35] who also obtained DRDFs for molten PE from WAED. They criticized the earlier results of Ovchinnikov and co-workers as having considerable termination errors and inadequate background corrections. Their resultant DRDF, presumably with better corrections, shows three intramolecular peaks in the 1-3 $\AA$ region and one broad weak peak in the 4-6 A region. The broad peak was attributed to a distribution of intermolecular distances of about $5 \AA$ rather than any distance resulting from parallel chain segments. We do not know how they had avoided the difficulties generally encountered in WAED such as radiation damage and the problem of correction for inelastic scattering in the electron intensity data.

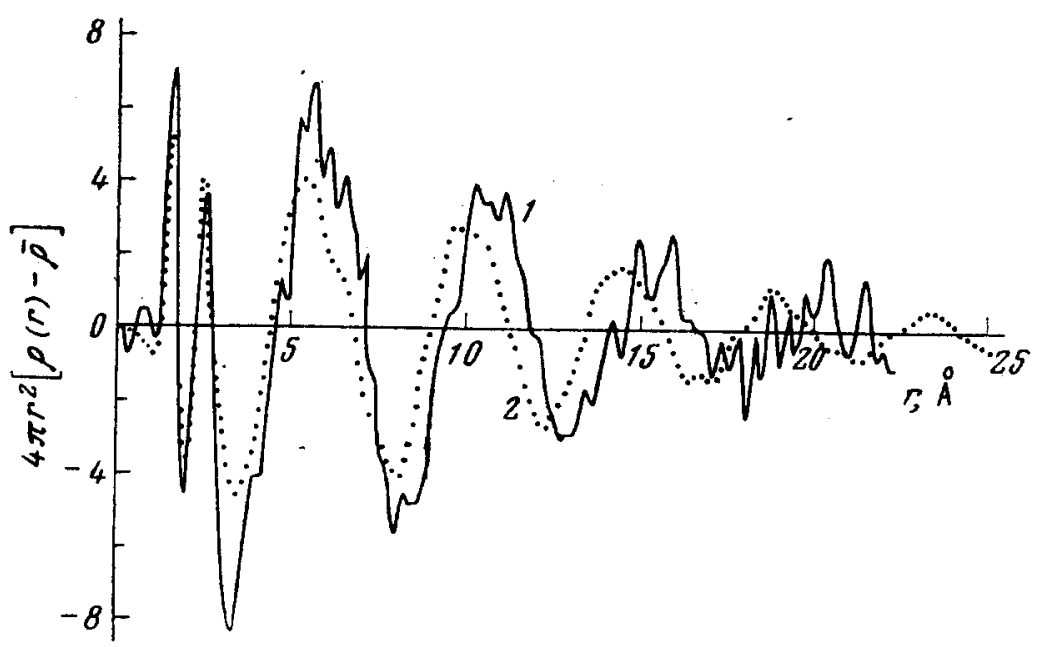

FIG. 10. DRDF for molten PE [35] v8. amorphotized PE [21].

In view of the above controversy, Longman et al. [35] have performed another DRDF study, but this time they based their analysis on the more reliable WAXS intensity data. They found broad intermolecular peaks at $\sim 5,10$, and $15 \AA$, and possibly one more at $\sim 20 \AA$, in addition to the common intramolecular peaks (shown by the continuous curve in Fig. 10). They concluded that for PE melt lateral order exists.

Recently we have applied DRDF analysis to WAXS of crystallized PE that has been made amorphous by $\gamma$ radiation [21]. In addition to the application of the separation method, we wanted to see if the paracrystalline theory can be applied to the DRDF of such a relatively simple polymeric system also, especially 
in view of the fact that upon radiation the simple orthorhombic lattice in PE changes to a simple hexagonal lattice before complete destruction of crystallinity.

Our corrected dotted DRDF is also shown in Fig. 10, together with the DRDF curve obtained from WAXS of the molten PE at $160^{\circ} \mathrm{C}$ by Wignall et al. Both curves show two intramolecular peaks at 1.5 and $2.5 \AA$. However, in the molten PE only four intermolecular peaks $(\sim 5,10,15$ and $20 \AA)$ are present as compared
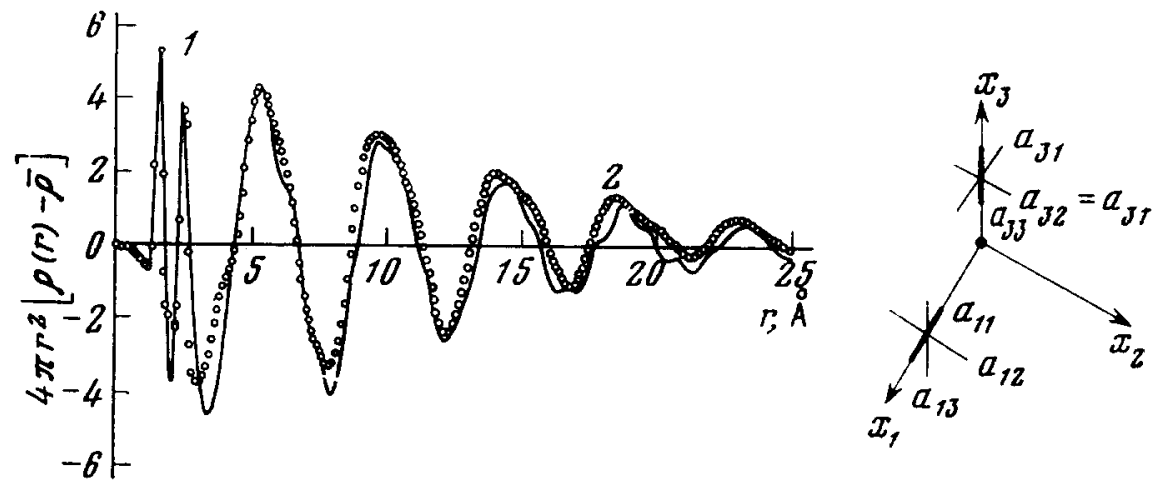

Fig. 11. Experimental vs synthetic DRDFs for amorphotized PE [21].

to five $(5,10,14,18$ and $23 \AA)$ seen in APE. In addition, the intermolecular peaks of the molten PE are broader, as expected, and shifted to higher $r$. The two curves are rather similar, both showing a periodicity of $\sim 5 \AA$. Application of separation method indicates that the peaks at $5 \AA$ or above are almost totally intermolecular in origin. Application of the paracrystalline lattice theory indicates that there are indeed ordered chain segments arranged in distorted lattices with lattice constants and distortion parameters given in Table 3. The two

Table 3. Distortion parameters and their sensitivity to "sUm"

\begin{tabular}{c|r|r|r|r|r}
\hline Parameters & \multicolumn{1}{|c|}{$a_{11}$} & \multicolumn{1}{|c|}{$a_{12}$} & $a_{13}$ & $a_{31}$ & \multicolumn{1}{c|}{$a_{33}$} \\
\hline Value, $\AA$ & 0.90 & 0.83 & $0 \cdot 15$ & 0.25 & $0 \cdot 10$ \\
$g^{*}, \%$ & 1 & 20 & 6 & 6 & 10 \\
Change in $g, \%$ & 1 & 2 & 2 & 2 & 2 \\
Increase in sum, \% & 50 & 10 & 3 & 5 & 10 \\
\hline
\end{tabular}

- With respect to $a=4.94$ and $c=2.54$ in hexagonal lattice.

most significant distortion parameters are $g_{11}=18 \%$ and $g_{33}=10 \%$. The $g_{38}$ distortion along the chain direction is expected to be smaller than $g_{11}$ because of the higher covalent forces in the chain direction as compared to the weaker intermolecular forces.

Based on this study we can say that a distorted hexagonal lattice is retained in APE. In the distorted 3-dimensional structure the atomic density is not dis- 
tributed uniformly in all directions but is concentrated in the direction of the nearest neighbours. Based on the similarity of the two curves shown in Fig. 11, we suggest that a distorted hexagonal lattice is probably also present in molten $\mathrm{PE}$. In such a distorted paraerystalline lattice, long range order is eventually lost at higher distances. Ordering is limited to $r=25 \AA$ for the molten PE. The effect of crosslinking is to introduce local distortions in the form of kinks as is the effect of temperature.

\section{CONCLUDING REMARKS}

It is much too early to assess the significance of the results from these DRDF studies. Like the earlier findings from electron microscopy, clearly more work is needed before the implications of such structural information can be fully understood. However, the challenge to take on a structural and relatively new approach to understand the behavior of a very important class of amorphous materials is clearly there, not only because of the existing amorphous myth about them but also because the present approaches based on either statistical calculations or average free volume measurements, etc. are not adequate in providing answers for their behaviors as non-Newtonian viscosity, memory effects, yielding, cold flow, crystallization and crosslinking, etc.

The author is pleased to acknowledge the financial support provided by the National Science Foundation (Polymers Program, Division of Materials Research, DMR74-00801) and the many lively discussions with Prof. Rolf Hosemann on the application of his paracrystalline theory to DRDF analyses.

\section{REFERENCES}

1. G. S. Y. YEH, J. Pure Appl. Chem. 31: 65, 1972

2. G. S. Y. YEH, J. Macromol. Sci. (Phys.) B6: 451,1972

3. G. S. Y. YEH and S. L. LAMBERT, J. Macromol. Sci. (Phys.) B6: 599, 1972

4. D. G. H. BALLARD, J. SCHELTEN and G. D. WIGNALL, Eur. Polymer J. 9: 965, 1973

5. J. SCHELTEN, G. D. WIGNALL and D. G. H. BALLARD, Polymer 15: 682, 1974

6. J. SCHLETEN, G. D. WIGNALL, D. G. H. BALLARD and G. W. LONGMAN 18: 111 , 1977

7. G. LIEGER, W. E. FISCHER and K. IBEL, J. Polymer Sci. (Phys.) B13: 39, 1975

8. G. ALLEN and T. TANAKA, Polymer 19: 271, 1978

9. G. S. Y. YEH, Kyoto Polymer Colloquium, 71-74, 1977

10. C. Clement and P. BOTHOREL, J. Chem. Phys. 61: 878, 1964; P. BOTHOREL, J. Colloid Interf. Sci. 27: 529, 1968

11. M. DE TTENMAIER and E. W. FISCHER, Kolloid Z. Polym. 251: 922, 1973

12. G. D. PATTERSON and P. J. FLORY, J. Chem. Soc. Faraday Trans. 2: 68, 1098, 1972

13. A. E. TONeLLI, J. Chem. Phys. 53: 4339, 1970

14. R. GenanNT, W. PeChHold and H. P. Grosmann, Colloid and Polymer Sci. 255:

15. C. S. 1977

285, WANG andG. S. Y. YEH, J. Macromol. Soi. (Phys.) B15: 107, 1978 
16. M. GUPTA and G. S. Y. YEH, J. Macromol. Sci. (Phys.) B15: 119, 1978

17. A. BJORNhaUg, O. ellefsen and B. A. TONNESEN, J. Polymer Sci. 12: 621, 1954

18. G. D. WIGNALL and G. W. LONGMAN, J. Mat. Sci. 8: 1439, 1973

19. S. M. WECKER, T. M. DAVIDSON and J. B. COHEN, J. Mat. Sci. 7: 1249, 1970

20. R. HOSEMANN, G. WILLMANN and B. ROESSLER, Phys. Rev. 6A: 2243, 1972; B. STEFFEN and R. HOSEMANN, ibid, 13B: 3232, 1976

21. M. GUPTA and G. S. Y. YEH, Submitted to J. Macromol. Sci. (Phys.), accepted.

22. P. HARFGeT and S. M. AHARONI, J. Mrcromol. Sci. (Phys.) B12: 209, 1976

23. J. R. KATZ, Z. Phys. Chem. A125: 321, 1927; Trans. Faraday Soc. 32: 77, 1936

24. G. S. Y. YEH, J. Macromol. Sci. (Phys.) B6: 465, 1972

25. G. S. Y. YEH and P. H. GEIL, J. Macromol. Sci. (Phys.) B1: 235, 1967

26. G. W. LONGMAN, R. P. SHELdoN and G. D. WIGNALL, J. Mat. Sci. 11: 1339, 1976

27. C. S. WANG and G. S. Y. YEH, Polymer 18: 1085, 1977

28. W. J. JACKSON, Jr. and H. F. KUHFUSS, J. Polym. Sci. (Chem.) 14: 2043, 1976

29. T. E. BRADY and G. S. Y. YEH, J. Polym. Letters 10: 731, 1972

30. E. B. BOKHYAN, Yu. K. OVChINNIKOV, G. S. MARKOVA and V. A. KARGIN, Polymer Sci. U.S.S.R. 13: 2026, 1971

31. D. LUCH and G. S. Y. YEH, J. Appl. Phys. 43: 4326, 1972

32. C. S. WANG and G. S. Y. YEH, (to be published)

33. G. S. Y. YEH, Critical Reviews in Macromol. Sci. 1: 173, 1972

34. J. K. OVCHINNIKOV, G. S. MARKoVA and V. A. KARGIN, Polymer Sci. U.S.S.R. 11: 329,1969

35. I. VOIGT-MARTIN and F. C. J. MIJLHOFF, J. Appl. Phys. 46: 1165, 1975

36. G. W. LONGMaN, C. D. WIGNaLL and R. P. SHELdoN, Polymer 17: 486, 1976

\title{
SOME SYNTHETIC POLYMERS WITH FUNCTIONAL GROUPS FOR BIOMEDICAL APPLICATIONS*
}

\author{
Ya. KaLaL \\ Institute of Macromolecular Chemistry, Czechoslovakian Academy of Sciences
}

Three groups of hydrophilic, soluble and biocompatible polymers (poly(hydroxyalkyl acrylates), $\mathrm{N}$-substituted polyacrylamides and polyamides of the polypeptide type) are examined as carriers of various functional groups. Tests showed that polymeric derivatives formed between the original functional groups and pharmacologically active groups possess biological activity. Fluorescent tracers were used to follow

* Vysokomol. soyed. A21: No. 11, 2447-2456, 1979. 